\title{
Medical Laboratory Disciplines: Time for Academic Reform and Structural Organization
}

\author{
A.K. Mukhopadhyay, MD* \\ Department of Laboratory Medicine, All India Institute of Medical Sciences, New Delhi, India.
}

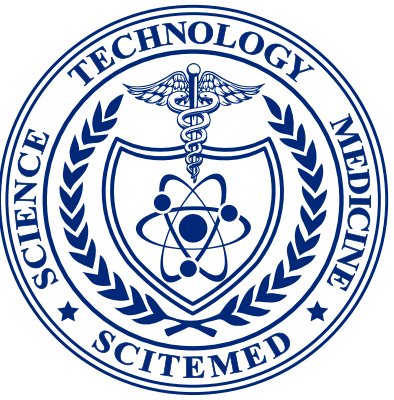

"Olothing is more powerful than an idea whose time has come"

Victor Hugo

boratory medicine is understood differently in different countries. Pathology is oftentimes used as the generic name for all laboratory-related medical disciplines. However, it should be understood that laboratory medicine is synonymous with clinical pathology also referred to as fluids medicine, which also includes chemical pathology, laboratory hematology, toxicology, transfusion medicine, microbial pathology, and biochemical metabolism among others. Laboratory medicine is also an example of a "smart lab" paradigm that encompasses robotic automation, quality assurance and microscopy, and other related outputs. These are oriented more towards providing key patient-centric service and dealing with investigations of immediate relevance with the shortest possible turn-around-time (TAT). This collective expertise serves as a type of "medical detective", and "laboratory consultant" of the clinical consultants.

With the shift of generic nomenclature from pathology to laboratory medicine, the latter becomes inclusive of all laboratory-related medical disciplines as outlined above. This shift of focus has provided an opportunity for academic and organizational reforms of different laboratory-related medical disciplines.

Any medical discipline has three objectives: service to patients, service to students (academics), and service to science (research and publications). Laboratory medicine is not an exception to this. Rather, laboratories serve as the "backbone" of evidence-based medicine and the "jugular vein" of a hospital. The service to students (education) helps generation of expert resources at the post-graduate and post-doctoral super-specialty levels of laboratory science.

The disciplines of pathology, microbiology and biochemistry have a great role in concept building for medical undergraduates. However, the significance of what is being done in a hospital's clinical laboratory for moment-to-moment management of patients is often not appreciated by the medical graduates. Postgraduate courses in general pathology offers knowledge and experience in anatomic pathology, histochemistry/cytochemistry and molecular diagnosis. Postgraduate training in biochemistry has embraced metabolomics, proteomics, genomics and epigenomics, while postgraduate training for medical microbiology ranges from bacteria to parasites, fungus to virus and bacteriophage, and their diagnosis in human conditions at cultural, biochemical, serological and molecular levels. Pathology, microbiology and biochemistry all teach immunology and molecular biology in the context of their respective subject matter. Strikingly, outside Unites States and across the globe, there are few postgraduate courses, which exclusively deals with holistic approaches to diagnostic patient care with appropriate training in automation, microscopy, accreditation, equipment and reagent procurement, and total quality management including safety, ethics and legal practice. There is an apparent need for the bolstering of laboratory medicine as a basic training requirement in postgraduate medical disciplines, worldwide.

To this end, in India and Bangladesh laboratory medicine has emerged as an independent postgraduate medical discipline. India's premier medical institute at Delhi, AlIMS, founded this discipline in 1988 and started a post-graduate course with a three-year residency program since 1997. Bangladesh took a similar approach, and following this example in India, Bangabandhu Sheikh Mujib Medical University (BSMMU) established this independent discipline in 2014. Bangladesh in 2017 has started replacing their two-year diploma courses, Master in Philosophy degree in clinical pathology with three-year degree course in laboratory medicine. This bore out as a result of country's service needs. Laboratory medicine for countries such as India, Bangladesh and even Canada (where it is called "general pathology") is a diagnostic service facility managed by laboratory physicians, who are postgraduates in laboratory medicine. The arrangement is useful to deliver integrated diagnostic services in the area of clinical pathology, laboratory hematology, microbiology, clinical biochemistry and rapid molecular tests, ensuring quality results with minimum turn-around-time in their respective vast rural and semi urban areas. The palpable gains are in economizing the realities of space, time, manpower, knowledge and budget cost centers. This arrangement offered a new option for students as well as for teachers. At a higher level, this also expanded the opportunity for the basic departments of pathology, microbiology and biochemistry to develop their respective post-doctoral fellowship courses such as neuro-, renal-, dermato- and gastrointestinal pathology, hematology, virology, parasitology, nanomedicine, genetics as well as, immuno-, and molecular medicine etc.

Having recognized the need of such academic orientation in laboratory-related disciplines, organizational reform of the laboratories in various teaching medical institutions and hospitals automatically fell into its natural place in the medical architecture. A representative diagnostic block would consist of three tiers. Tier I includes the central hospital laboratory (laboratory medicine) with a central phlebotomy facility and blood bank services (transfusion medicine). Like laboratory medicine, transfusion medicine is also poly-disciplinary in nature and caters to all outpatient and in-patient transfusion needs. Transplantation and reproductive medicine laboratories could provide specialized divisions of laboratory medicine. Tier II would consist of the laboratories in three basic disciplines: anatomic pathology, microbiology and biochemistry. Tier III laboratory services of a hospital in any medical institution would consist of a common research facility with all high-end equipment (for example, mass spectroscopy, next generation sequencing, LC-MS, GC-MS ICP-MS, confocal microscopy, cell sorters, molecular tracer, image analyzer, stem cell and organoid research facility etc.).

TAT in a Tier I system varies from a few minutes (in case of point of care) to a few hours; in Tier II, 12-72 hours; and in Tier III, usually a week. Tier I laboratories work round-the-clock; Tier II laboratories, from 9 AM to 5 PM; while a Tier III laboratory, although always accessible, mostly works when needed. The Tier I laboratory is run by laboratory physician, Tier II by respective postgraduates and consultants, and Tier III by laboratory scientists with Ph.D. degree. Laboratory scientists (Ph.Ds) have a greater role in Tier III laboratories on which specialty training mostly depends. The scope in Tier III is unlimited and includes basic cell biology, synthetic biology, biologically inspired systems science (BISS), bio-robotics and personalized medicine. None of the tiers are absolute or exclusionary and they may interact with each other for a common purpose. Integrated laboratory and clinical information systems serves to unify patients and holistic training of the residents, availing many opportunities for 


\section{EDITORIAL}

inter-laboratory and laboratory-clinician dialogues as well as generating revenues for the hospital, research papers for the institution, and shared experts for the nation.

Last, but not the least, medical laboratories cannot be run without trained technologists. Therefore, their training courses should be organized accordingly in undergraduate (B.Sc.), postgraduate (M.Sc.) and specialty level (Ph.D.) with corresponding appointment in Tier I to III. Such an approach can serve to herald in the next decade as the decade of the laboratory by fostering the structural and academic organization for the discipline of medical laboratory science and avail its laboratory physicians their due credit. The Journal of Laboratory Medicine provides global access to the maturation of this discipline and is poised to help beckon in such a future.

\section{Article Information}

*Correspondence: A.K. Mukhopadhyay, MD

Department of Laboratory Medicine, All India Institute of Medical Sciences, New Delhi-110029, India.

Email: Mukhoak1953@gmail.com

Received: Aug. 17, 2017; Accepted: Aug. 23, 2017; Published: Sep. 19, 2017

DOI: 10.24983/scitemed.jacp.2017.00032

Copyright $\odot 2017$ The Author. This is an open-access article distributed under the terms of the Creative Commons Attribution 4.0 International License (CC-BY). 